\title{
A Novel Particle Swarm Optimization With Genetic Operator and Its Application to TSP
}

\author{
Bo Wei, Zhejiang Sci-Tech University, China \\ Ying Xing, East China Jiaotong University, China \\ Xuewen Xia, Minnan Normal University, China \\ iD https://orcid.org/0000-0002-4938-1479 \\ Ling Gui, Minnan Normal University, China
}

\begin{abstract}
To solve some problems of particle swarm optimization, such as the premature convergence and falling into a sub-optimal solution easily, the authors introduce the probability initialization strategy and genetic operator into the particle swarm optimization algorithm. Based on the hybrid strategies, they propose an improved hybrid particle swarm optimization, namely IHPSO, for solving the traveling salesman problem. In the IHPSO algorithm, the probability strategy is utilized into population initialization. It can save much more computing resources during the iteration procedure of the algorithm. Furthermore, genetic operators, including two kinds of crossover operators and a directional mutation operator, are used for improving the algorithm's convergence accuracy and population diversity. At last, the proposed method is benchmarked on nine benchmark problems in TSPLIB, and the results are compared with four competitors. From the results, it is observed that the proposed approach significantly outperforms others on most of the nine datasets.
\end{abstract}

\section{KEYWORDS}

Cognitive Computing, Deterministic, Genetic Algorithm, Genetic Operators, NP-Complete Problem, Particle Swarm Optimization, Probabilistic, Travel Salesman Problem

\section{INTRODUCTION}

The travel salesman problem (TSP) firstly formulated as a mathematical problem in 1930 is a typical NP-complete problem. In recent years, it attracts much attention in mathematics and computation sciences communities and has been one of the most intensively studied problems in optimization(Bello, Gomez, Nowe, et al, 2007). The purpose of the TSP is to find the shortest journey, which each city can be visited only once and returns to the starting city. Concretely, it means to search for an arrangement of natural subsets (the elements of $\mathrm{C}$ are the serial number of cities). The TSP can be described as following:

$$
T_{d}=\sum_{d=1}^{n-1} d_{c_{d} c_{d+1}}+d_{c_{n} c_{1}}
$$


where $d_{c_{d} c_{d+1}}$ represents the distance between city $c_{d}$ and city $c_{d+1}$.

Since meta-heuristic methods are able to find solutions rapidly by the strong ability of exploring and exploiting the full search space, those algorithms have attracted a lot of attention in solving global optimization problems ( $\mathrm{Li}$, Wang, 2019; $\mathrm{Li}$, Chen, et al, 2018). Considering the advantage of it, many heuristics algorithms are applied to deal with TSP in recent years, such as simulated annealing (SA) (Li, Zhou, Zhang, 2011), tabu search (TB)(Lin, Bian, Liu, 2016), neural networks (NN)(Li, Qiao, Li, 2016), and genetic algorithm (GA)(Pang, Wang, Zhou, et al, 2004; Li, Liang, et al, 2019).

Particle swarm optimization (PSO) proposed by Kennedy and Eberhart(Kennedy, Eberhart, $1995)$ in 1995 is an effective approach to address continuous problems. In recent years, PSO has been successfully applied in many optimization problems, such as function optimization(Yin, Li, Gao, et al, 2016, Wang, Zhang, Li, et al, 2018), wavelet mutation(Ling, Iu, Chan, et al, 2008), image processing (Chandramouli, Izquierdo, 2006), and automatic control(Chatterjee, Pulasinghe, Watanabe, et al, 2005; Chen, Ren, Fan, 2006). However, the conventional PSO cannot directly be applied in TSP which is a type of discrete problem rather than a continuous problem. So the PSO method is proposed to solve TSP firstly by Clerc(Clerc, 2004) in 2004. Then Hendtlass(Hendtlass, 2003) uses PSO to solve small-size TSP by adding a memory capacity for each particle. Pang(Pang, Wang, Zhou, et al, 2004) combined the characteristics of PSO with the concept of fuzzy theory.

Based on the previous research, an improved hybrid PSO (IHPSO) is proposed in this work, in which some new features are introduced to improve its comprehensive performance. Firstly, a probability initialization is used to reduce the complexity as well as to achieve better performance on a large scale in TSP. Relying on the strategy, the initial population can obtain the guidance of prior knowledge. Secondly, two different matrices are introduced to enhance two crossover operators. Through two crossover operators the algorithm can take full advantage of the global best solution Gbest and the personal best solution Pbest. Thirdly, a directional mutation operator is proposed to avoid the excellent genes broken, thus to improve its solution accuracy.

The rest of this paper is organized as following. Section 2 describes the framework of the canonical PSO. The description of TSP is shown in Section 3. The hybrid particle swarm optimization is used for discrete problems is mentioned in Section 4. Details of IHPSO are described in Section 4. And the experimental results between IHPSO and other 4 test functions in 9 benchmark problems in TSPLIB(Reinelt, 1991) are detailed in Section 5. Finally, conclusions are provided in Section 6.

\section{PSO}

\section{Canonical PSO}

PSO is typical swarm intelligence algorithms inspired by birds' predatory behaviors. At the beginning of PSO, a group of particles is initiated in a feasible space, and each particle represents a penitential solution of an optimization problem. During the searching process in hyperspace, the $i$ th particle is associated with two vectors, i.e., a position vector $\mathrm{Xi}=\left[\mathrm{x}_{\mathrm{i} 1}, \mathrm{x}_{\mathrm{i} 2}, \ldots, \mathrm{x}_{\mathrm{iD}]}\right.$ and a velocity vector $\mathrm{Vi}=$ $\left[\mathrm{v}_{\mathrm{i} 1}, \mathrm{v}_{\mathrm{i} 2}, \ldots, \mathrm{v}_{\mathrm{iD}}\right]$, where $D$ represents the dimensionality of an objective problem. The vector $\mathrm{X}_{\mathrm{i}}$ can be regarded as a candidate solution to the problem while the vector $\mathrm{V}_{\mathrm{i}}$ denotes the search direction and step size of the $i$ th particle.

In each generation, the $i$ th particle updates its velocity and position relying on the global best solution Gbest $=\left[\mathrm{gb}_{1}, \mathrm{gb}_{2}, \ldots, \mathrm{gb}_{\mathrm{D}}\right]$ and its personal best solution Pbest $=\left[\mathrm{pb}_{\mathrm{i} 1}, \mathrm{pb}_{\mathrm{i} 2}, \ldots, \mathrm{pb}_{\mathrm{iD}}\right]$. The update rules are defined as following:

$$
v_{i j}^{t+1}=\omega \cdot v_{i j}^{t}+c_{1} \cdot r_{1} \cdot\left(p b_{i j}-x_{i j}^{t}\right)+c_{2} \cdot r_{1} \cdot\left(g b_{j}-x_{i j}^{t}\right)
$$


$x_{i j}^{t+1}=x_{i j}^{t}+v_{i j}^{t+1}$

where $\omega$ is an inertia weight that determines the influence of previous velocity; $c_{1}$ and $c_{2}$ are two numbers random distributed in the range of $[0,1] ; x_{i j}^{t}$ and $v_{i j}^{t}$ represent the $i$ th particle's position and velocity in the $j$ th dimension at generation $t$, respectively.

\section{Hybrid PSO for TSP}

The algorithm described above is regarded as the traditional PSO, which is very straightforward applied in continuous problems. However, for discrete problems, the traditional PSO doesn't work without modifications. Therefore, to solve the discrete problem, there have been attempts to modify the PSO algorithm.

Clerc proposed a hybrid PSO (HPSO) method for the discrete problem of TSP firstly(Clerc, 2004). In the research, some modified operators for update of the position and velocity in PSO are proposed to meet requirements of TSP, such as the opposite of velocity, the addition of position and velocity (movement), the subtraction of two positions, the subtraction and addition of two velocities, and the multiplication of velocity and constant. Nevertheless, the size of the TSP problems involved in the work is all smaller than 17. Hendtlass(Hendtlass, 2003) use PSO to solve small-size TSP by adding a memory capacity for each particle. The purpose of adding a memory capacity is used by the specific alternate target points instead of the current local best position. In this way, the efficiency and diversity of the algorithm are improved. Pang et al.(Pang, Wang, Zhou, et al, 2004) combined the characteristics of PSO with the concept of fuzzy theory, and each particle represents a fuzzy matrix. Fuzzy matrices are used to represent the position and velocity of the particles in PSO, meanwhile, the operators in the original PSO are redefined. Wang et al.(Wang, Huang, Zhou, et al, 2003) redefined operators of PSO by introducing the concepts of "swap operator" and "swap sequence". Therefore, TSP could be solved by the PSO in another way(Shaj, Akhil, Asharaf, 2016). Kennedy and Eberhart proposed a discrete binary version of PSO by defining particles' trajectories and velocities in terms of changes of probabilities that a bit will be in one state or the other proposed an improved version of PSO(Chang, 2005), which includes two phases. The first phase includes fuzzy C-means clustering, a rule-based route permutation, a random swap strategy, and a cluster merger procedure. Meanwhile, a genetic-based PSO procedure is used in the second phase(Chang, 2005). Shaj V. et al(Shaj, Akhil, Asharaf, 2016) add an edge recombination operator in update of the velocity operator. So that the search can be effectively directed to better corners of the solution space in each iteration.

Hybrid PSO abandons the function of tracking the utmost value to update the particle's position in the traditional PSO. In fact, hybrid PSO includes two crossover operators and a mutation operator into PSO. The process of breeding offspring based on the two crossover operators is shown in Figure 1.

The steps of hybrid PSO can be described as follows:

Step1: Parameter setting and population initialization.

Step2: Evaluate all individuals in the population.

Step3: Update the Pbest and Gbest according to the particles' fitness.

Step4: Perform the crossover operator between particles' and Pbest to get new particles.

Step5: Crossover operator between particles' and Gbest to get new particles.

Step6: The mutation operator of particles.

Step7: If the algorithm reaches termination conditions, the algorithm breaks off; or else, back to Step2.

\section{IHPSO for TSP}

The HPSO introduced above is a simple superposition of PSO and genetic operator. With the modification, the PSO variant can be directly used in discrete problems. However, its convergence 
Figure 1. The process of breed offspring in hybrid PSO

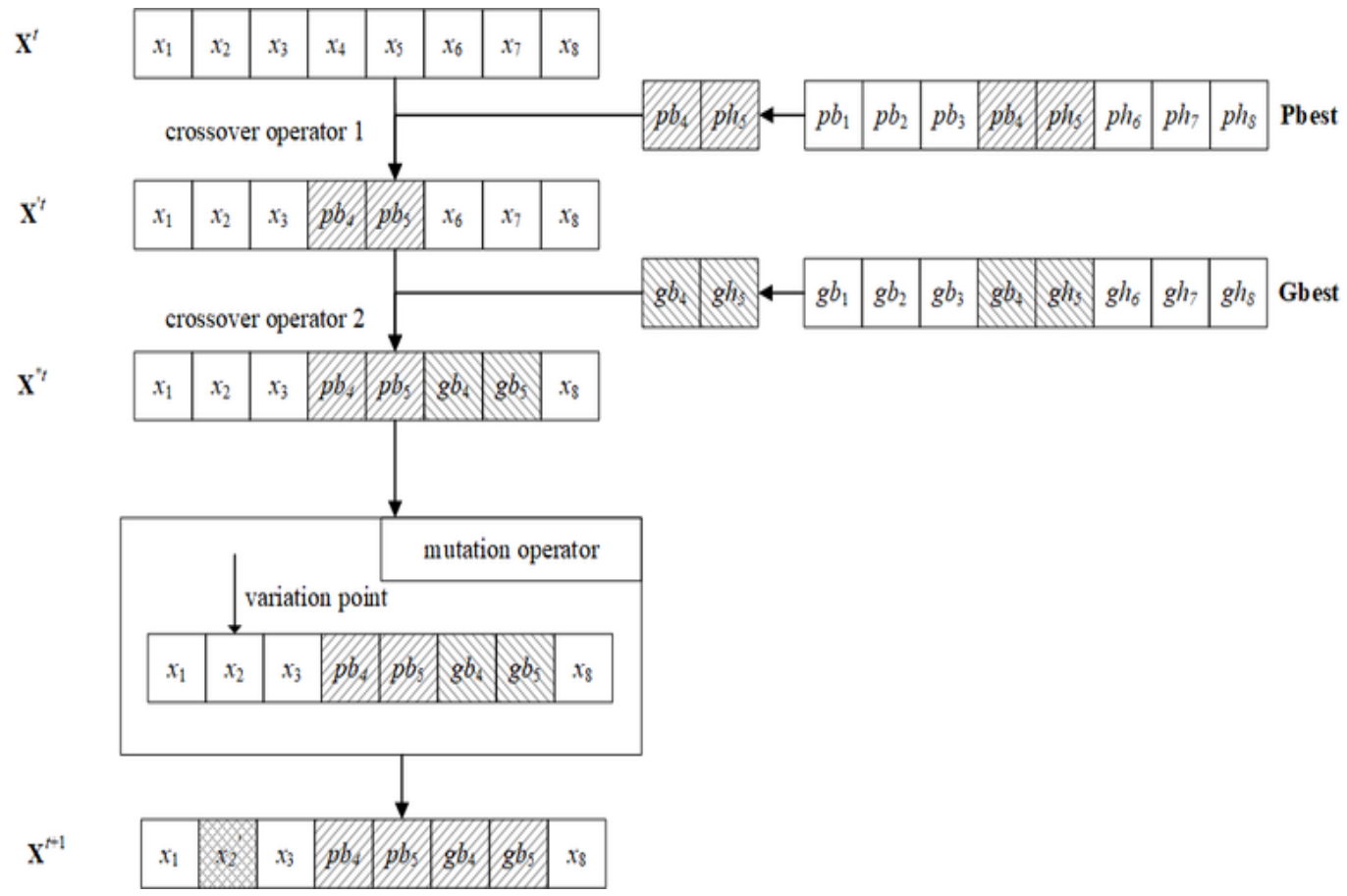

speed is not high enough. In other words, the original hybrid PSO is easy to fall into a locally optimal solution. In order to improve the algorithm performance in TSP, we propose an IHPSO, in which there are four novel strategies.

Considering there are distinct characteristics of Gbest and Pbest, we proposed two different crossover operators to take full advantage of them, respectively. Concretely, a deterministic crossover operator and a deterministic crossover operator are conducted between a particle between Gbest and Pbest, respectively. In this case, the population can achieve a trade-off between convergence speed and diversity. Moreover, a probabilistic matrix (pcitylink) and a deterministic vector (dcitylink) are established for the crossover operator in this algorithm.

The details of new strategies and the flowchart of IHPSO are presented as follows one by one.

\section{Probability Initialization}

Although a random initialization method, which is very popular in the PSO community, can increase the diversity of the initial population, it ignores much helpful guidance information that can be extracted from cities' coordinates. Hence, the aimless initialization method has lower efficiency, especially in large scale TSP. In this research, a new initialization mode is proposed to overcome the above-mentioned shortcomings.

To takes advantage of some prior knowledge based on the cities' positions, an adjacency matrix is constructed as (4).So that individuals in the initial population have very high fitness: 


$$
\text { citydist }=\left[\begin{array}{l}
d_{11}, d_{12}, \ldots, d_{1 n} \\
d_{21}, d_{22}, \ldots d_{2 n} \\
\ldots \ldots \ldots \ldots \ldots \ldots . . . \ldots \ldots \\
d_{n 1}, d_{n 2}, \ldots, d_{n n}
\end{array}\right]
$$

where $\mathrm{d}_{\mathrm{ij}}$ denotes the distance between the $i$ th city and $j$ th city.

Based on the matrix citydist, a heuristic initialization parameter matrix can be defined as (5):

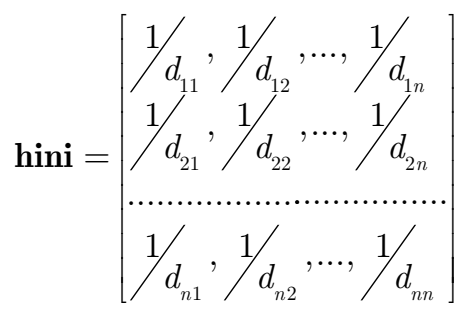

The key idea of the probability initialization is that the initialization of the population is based on a transfer probability matrix, an element in which indicates the probability that from the current city to the next city. Its formula is as (6):

$\mathbf{P}(v)=\operatorname{hini}(i, v)^{\alpha}$

or:

$\mathbf{P}=\left(p_{1}, \ldots, p_{v}, \ldots, p_{k}\right)$

where $\alpha=5 ; k=$ length (visiting), $0 \leq k \leq(n-1)$ visiting is the visiting cities vector. hini is the heuristic initialization parameter matrix, and the size of hini is $n^{*} n$ ( $n$ is the number of cities). Thereinto, $i$ represents a serial number of the last one of visited cities, and $j$ represents a serial number of all the visiting cities. In this way, $\mathbf{P}(v)$ represents the probability between the last one of visited cities $i$ and the visiting cities $v$. With the initialization process going on, the scale of visiting is getting smaller and smaller. In other words, the size of $\mathbf{P}$ is decreasing from $(n-1)$ to 0 with the initialization process.

After the calculation of the transition probability, we need in order to select which city we should shift next. At this moment, each visiting city is given a ratio, and this ratio is the proportion of this city in entire visiting cities. As the algorithm progresses, the number of cities that need to be visited will gradually decrease, then the ratio will change. An additive approach is used for the final probability selection matrix. In the end a random number is generated as a criterion for choosing the next city to visit.

Built on the above description, the strategy of probability initialization can be detailed as Algorithm 1. 
Algorithm 1. Probability initialization

01: For $j=2: n / / n$ is the number of cities

02: For $i=1: p s / / p s$ denotes the population size

03: Build visited cities vector visited, visiting cities vector visiting, and

the selection probability of visiting cities Vector $\mathbf{P}$;

04: Update visiting according to the data in visited;

05: For $\mathrm{k}=1$ :length(visiting) //calculate the probability of being

// selected for each candidate city

06: $\mathbf{P}(\mathrm{k})=\mathbf{T a}(\operatorname{visited}(\mathrm{end}), \text { visiting }(\mathrm{k}))^{\wedge} \alpha$;

07: End

08: $\mathbf{P}=\mathbf{P} /(\operatorname{sum}(\mathbf{P}))$;

09: Pcum=cumsum $(\mathbf{P})$

10: select $=$ find $($ Pcum $>=$ rand $)$;

11: $t o \_v i s i t=\operatorname{visiting}(\operatorname{select}(1))$;

12: individual $(\mathrm{i}, \mathrm{j})=$ to_visit // putting visited cities into individual;

//The complete individual is initial population

13: End

14: End

\section{Probabilistic Crossover Operator}

In PSO, Pbest of a particle retains some helpful personal experience. Thus, applying the Pbest to guide the particle has a positive effect during the optimization process. In order to efficiently utilize some favorable characteristics of the Pbest, we use the probabilistic crossover operator in this research.

The probabilistic crossover operator is on the basis of the probabilistic matrix pcitylink:

$$
\text { pcitylink }=\left[\begin{array}{l}
a_{11}, a_{12}, \ldots, a_{1 r} \\
a_{21}, a_{22}, \ldots ., a_{2 r} \\
\ldots \ldots \ldots \ldots \ldots \ldots . . . \\
a_{n 1}, a_{n 2}, \ldots, a_{n r}
\end{array}\right]
$$

The deterministic matrix pcitylink $=\left(\mathbf{A}_{1}, \mathbf{A}_{2}, \ldots, \mathbf{A}_{i}, \ldots, \mathbf{A}_{n}\right)(n$ is the number of cities, $i=1,2, \ldots$, $n$ ) stores a serial number of cities, and the size of this matrix is $n^{*} r$. And the $\mathbf{A}_{i}=\left(\mathrm{a}_{i 1}, \mathrm{a}_{i 2}, \ldots, \mathrm{a}_{i l}, \ldots\right.$, $\mathrm{a}_{i \mathrm{r}}$ ) represent information about $i$ th city. Furthermore, $l$ denotes the $l$ th nearest city for the $i$ th city. For example, $\mathrm{a}_{i l}$ represents the $l$ th nearest city from $i$ th city. 1 to $r$ indicates the distance between two cities from near to far.

The probability is reflected in the selection of the gene's cros insertion position, which is collected from Pbest. Matrix pcitydistlinkcum represents the probability of the corresponding city transfer to 


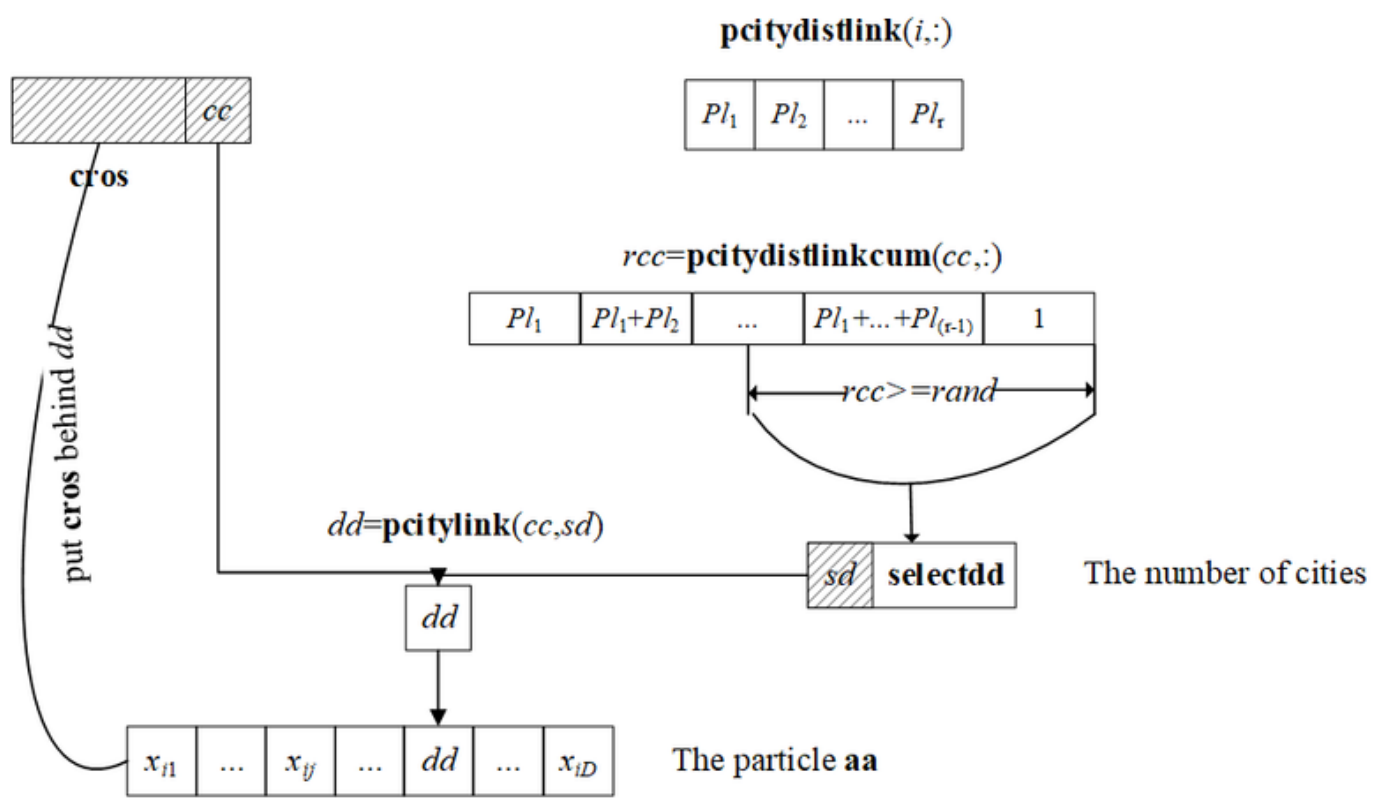

the next one. The uniqueness of this matrix is that each element is the sum of itself and the element in front of him in pcitydistlink. pcitydistlink is a distant probability matrix. According to the last element of cros, the corresponding element $r c c$ in pcitydistlinkcum can be selected. By comparing the numerical values of $r c c$ and random number rand, a range of insertion positions selected can be got from pcitylink. The element of pcitylink is the ordering information from near to far, so that we choose the first element of selected as an insertion point of gene fragment cros. We put cros on the back of $d d$. There is a special case here. The same elements in current particle aa and cros are previously deleted, so if the $d d$ in the element of cros, we cannot find $d d$ in aa. In this case, gene cros will be putting at the last aa.

The above expression can be represented by Fig. 2 and Fig. 3 while the pseudo code of it is presented in Algorithm 2.

If $d d$ is inside cros, the probabilistic crossover operator can be showed as Fig. 2

If $\mathrm{dd}$ is not inside cros, the probabilistic crossover operator can be showed as Fig.3.

\section{Deterministic Crossover Operator}

In PSO, Gbest is the global best-so-far position, and there is an only Gbest during the evolution of the algorithm. Compared to other particles, Gbest contains much more promising information, which can provide more effective knowledge for other individuals. This means that Gbest has a strong guiding significance for population evolution. For the characteristics of Gbest,a deterministic crossover operator is used to operate on Gbest.

The deterministic crossover operator is on the basis of the deterministic vector dcitylink:

dcitylink $=\left[m_{11}, \ldots, m_{i 1}, \ldots, m_{n 1}\right]^{\mathrm{T}}$

$n$ is the number of cities, $i=1,2, \ldots, n ; m_{i 1}$ represent the nearest city to city $i .1$ to $(n-1)$ indicates the distance between two cities from near to far. 


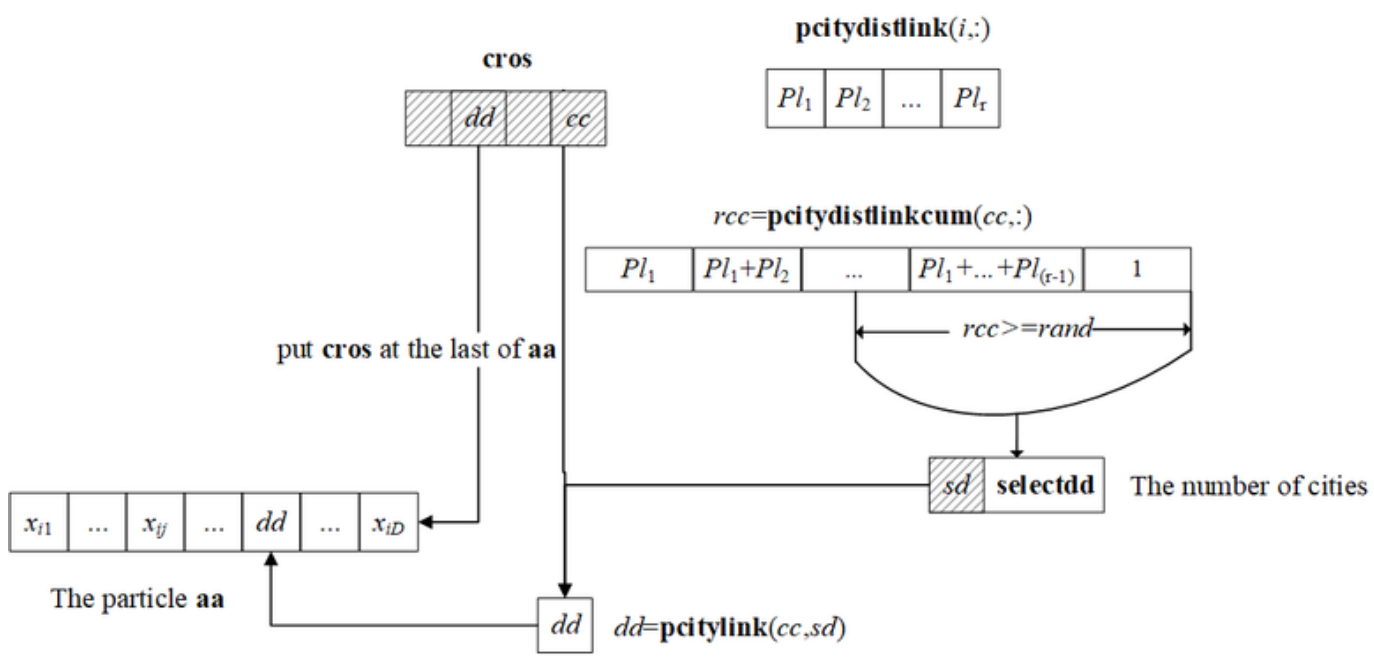

The deterministic crossover operator means the selection of the gene's cros insertion position is stationary. This kind of fixation doesn't mean the insertion position is changeless for each particle or in each generation. This means that the selection method is stationary. In simple terms, the gene cros is inserted behind the element which is the closest one to the first element of cros.

$c c$ is the first element of cros, and $d d$ is the closest element according to the vector citylink. Putting cros behind the $d d$ means the distance between $d d$ and $c c$ is the shortest. Consequently, it is a purposeful insertion, and better algorithm performance can be achieved. There is also a special case here. The same elements in current particle aa and cros are previously deleted, so if the $d d$ in the element of cros, we cannot find $d d$ in aa. In this case, gene fragment cros will be putting at the last aa.

The above process can be demonstrated by Figure 4 and Figure 5 while the pseudo-code of it is presented in Algorithm 3.

If $d d$ is inside cros, the deterministic crossover operator can be shown as Figure 4 .

Algorithm 2. Probabilistic crossover operator

\begin{tabular}{|l|}
\hline 01: Generate two intersection bits $\mathrm{c} 1$ and c2 on Pbest; \\
\hline 02: Generate gene fragment to be crossed cros; \\
\hline 03: Deleting the same part between current particle aa and cros; \\
\hline 04: cc=cros $(1) ;$ \\
\hline 05: rcc=pcitydistlinkcum(cc,:); \\
\hline 06: selectdd=find(rcc $>=$ rand); \\
\hline 07: dd=pcitylink(cc, sd); \\
\hline 08: If dd does not equal to the element of cros \\
\hline 09: Put cros on the back of dd; \\
\hline 10: Else \\
\hline 11: Put cros at the last of current particle aa; \\
\hline 12: End
\end{tabular}


Figure 4. Case 1 of deterministic crossover operator

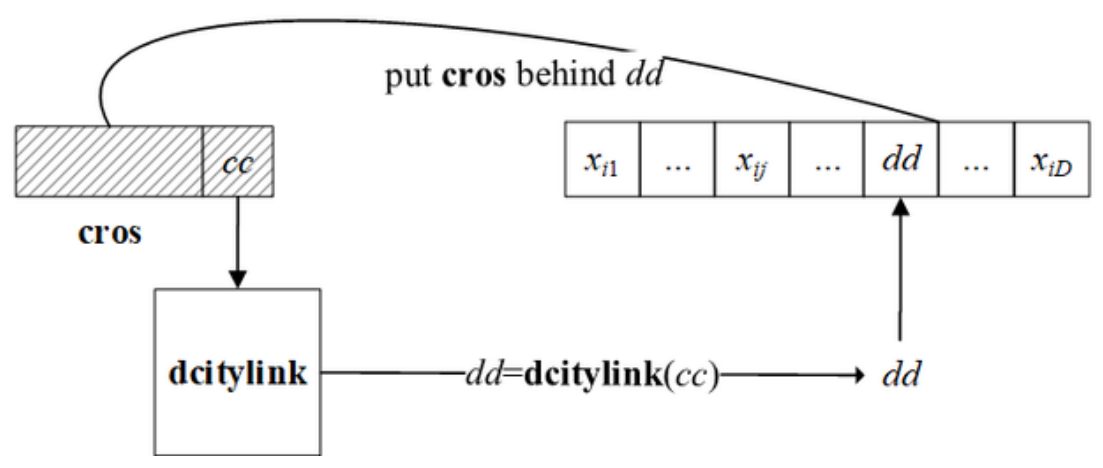

The particle aa

If dd is not inside cros, the deterministic crossover operator can be showed as Figure 5.

\section{Directional Mutation Operator}

Mutation operator can add a little disturbance to an individual, and then improve population diversity. Although a random mutation operator can increase population diversity to some extent, it may damage the excellent genes of those promising particles. Thus, to overcome the shortcomings of the random mutation operator, a directional mutation operator is proposed in this work. The main idea of the directional mutation operator is that change the data of the point behind the mutation point, and this change is on the basis of the matrix dcitylink. Specifically, $x x$ is the element of the mutation point in the current particle aa, and the closest element $c k$ can be found according to dcitylink. So the combination of $x x$ and $c k$ is the best for element $x x$. At last, the position of $c k$ in the aa should be found and replaced by temp. In this way, the algorithm achieves the directional mutation without any overlapping parts.

The above expression can be illustrated as Figure 6 while the pseudocode of it is presented in Algorithm 4.

Figure 5. Case 2 of deterministic crossover operator

\section{cros}

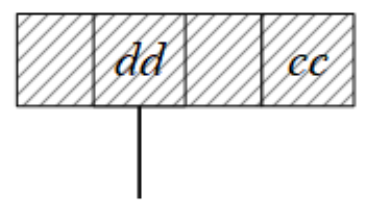

put cros at the last of aa

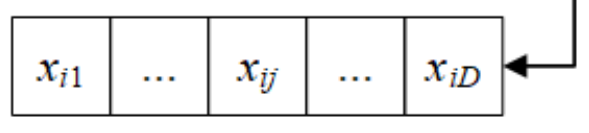

The particle aa 
Algorithm 3. Deterministic crossover operator

01: Generate two intersection bits c3 and c4 on Gbest;

02: Generate gene fragment to be crossed cros;

03: Delete the same part between current particle aa and cros;

04: $\mathrm{cc}=\operatorname{cros}(1)$;

05: $\mathrm{dd}=$ dcitylink $(\mathrm{cc})$;

06: If dd does not equal to the element of cros

07: Put cros on the back of $\mathbf{d d}$;

08: Else

09: Put cros at the last of current particle aa;

10: End

Figure 6. Directional mutation operator

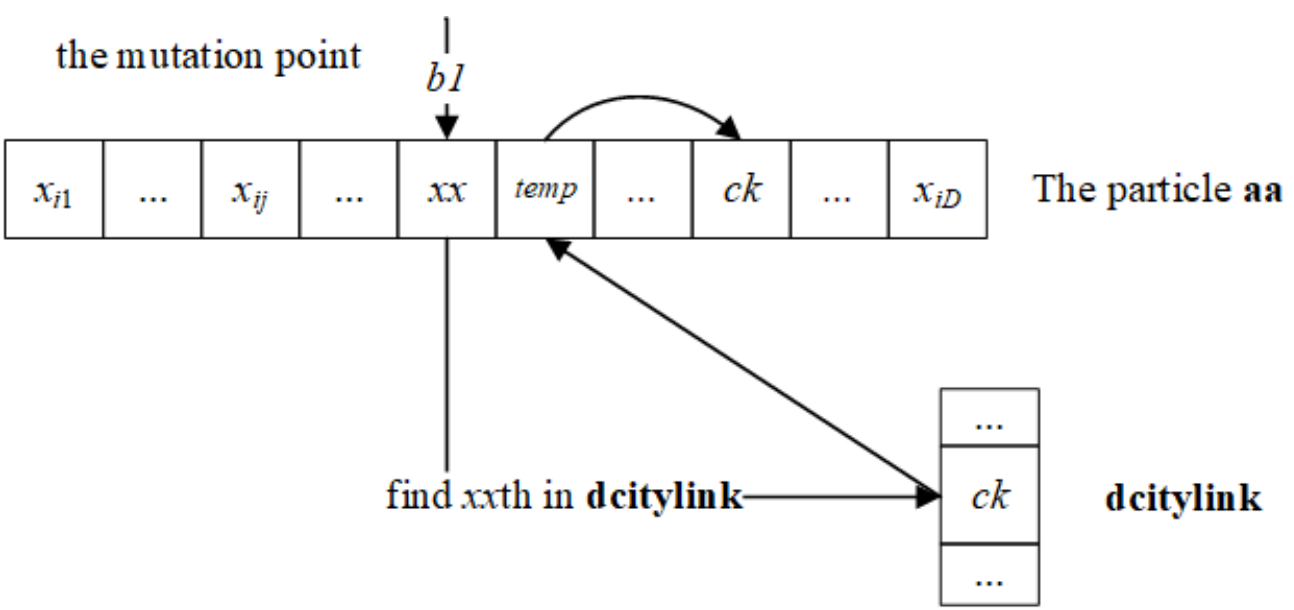

Algorithm 4. Directional mutation operator

01: Generate a mutation point randomly $b l$;

02: Take the element $x x$ at the mutation point and temp behind the mutation

operator from the current particle aa;

03: $c k=\operatorname{citylink}(x x)$

04: $\mathbf{a a}(b 1+1)=c k ; / / i$ repents the serial number of individual.

05: Find the position of $c k$, and taking place this position with temp 


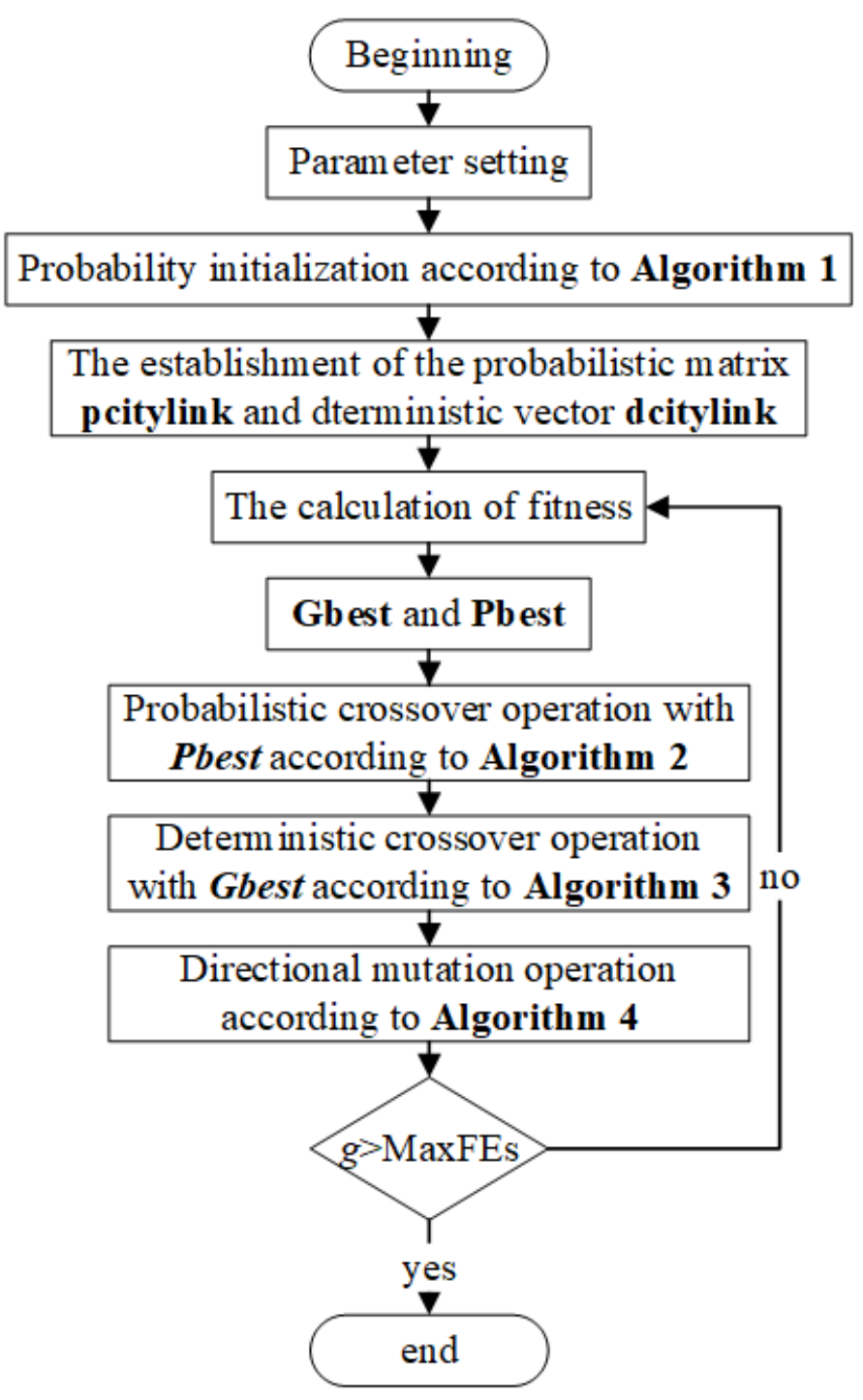

\section{IHPSO}

Based on the components introduced above, IHPSO can be described as shown in Figure 7 and Algorithm 5.

\section{EXPERIMENT RESULT AND ANALYSIS}

\section{Comparison of IHPSO and Peer Algorithms}

The comparison results between IHPSO and other 4 peer algorithms, in terms of mean value (Mean) and standard deviation (Std.Dev) of 30 independent runs, are listed in Table 1. In each row best results are bolded. Furthermore, the best tour paths for the 9 TSP achieved by IHPSO are demonstrated in Figure 8. 
Algorithm 5. IHPSO

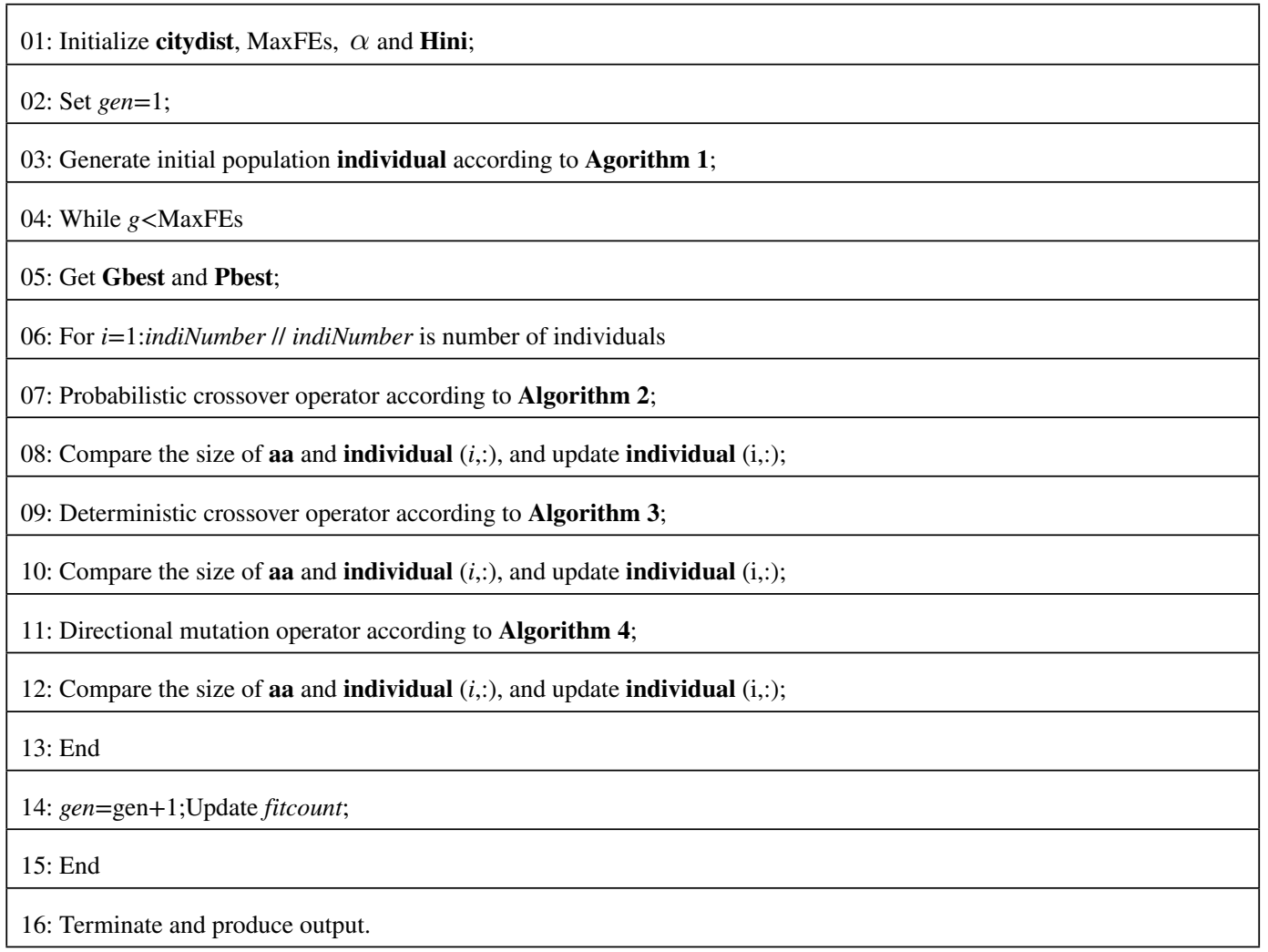

From the results listed in Table 1, we can see that IHPSO displays the best performance on all the test problems. Although IHPSO and HPSO belong to the PSO community, IHPSO dominates HPSO on all the 9 problems. Furthermore, with the increase in the cities' number, the advantages of IHPSO are becoming more and more obvious. Moreover, the time-consuming of those five methods are listed in Table 2. We can see from Table 2 that, IHPSO obtained second place in a time-consuming ranking. Thus, we can obtain a preliminary conclusion that IHPSO can yield very favorable performance on large scale TSP, and has promised scalability.

\section{Performance of Genetic Operators}

In order to examine the effectiveness of genetic operators (probability initialization, probabilistic crossover, deterministic crossover, and directional mutation operator) in IHPSO, we evaluate the performance of them by removing each one at a time. In Table 3, IHPSO(a) refers to the method removed probability initialization in IHPSO, IHPSO(b) refers to the method removed probabilistic crossover operator, IHPSO(c) refers to the method removed deterministic crossover operator, and IHPSO(d) refers to the method removed directional mutation in IHPSO. The performance of IHPSO, IHPSO(a), IHPSO(b), and IHPSO(d)methods for TSP problems can be seen in Table 3.

It can be seen from these results that IHPSO obtained the best results among five methods on all of the nine data sets. The positive effect of the genetic operators has been validated according to the results listed in Table 3. For example, for the data set ch130(ch150), the best result of IHPSO is 6372.3(7047.9), while the results of IHPSO(a), IHPSO(b), IHPSO(c), and IHPSO(d) are, respectively, reported 7131.40(7888.70), 6736.30(7428.60), 7563.30(8156.40), 6798.10(7322.50). According to 
Table 1. Comparison results $(\mathrm{km})$ between IHPSO and other 4 peer algorithms

\begin{tabular}{|c|c|c|c|c|c|c|}
\hline & & IHPSO & HPSO & GA & SA & TB \\
\hline \multirow{3}{*}{ eil51 } & Best & 428.87 & 441.84 & 446.34 & 461.79 & 447.76 \\
\hline & Mean & 448.35 & 463.52 & 467.37 & 503.01 & 476.90 \\
\hline & Std.Dev & 9.23 & 20.74 & 8.97 & 28.27 & 3.45 \\
\hline \multirow{3}{*}{ berlin52 } & Best & 7544.4 & 7716.7 & 7647.2 & 8645.3 & 8167.7 \\
\hline & Mean & 7938.49 & 8302.01 & 8259.24 & 9276.67 & 9238.71 \\
\hline & Std.Dev & 179.43 & 382.55 & 79.48 & 188.87 & 389.48 \\
\hline \multirow{3}{*}{ eil76 } & Best & $\mathbf{5 5 7 . 5 0}$ & 597.0247 & 620.83 & 627.66 & 595.46 \\
\hline & Mean & 581.66 & 650.95 & 687.31 & 703.76 & 654.20 \\
\hline & Std.Dev & 15.51 & 30.9 & 47.60 & 26.01 & 30.30 \\
\hline \multirow{3}{*}{ rat99 } & Best & 1249.8 & 1584.4 & 1850.9 & 1659.1 & 1516.4 \\
\hline & Mean & 1356.21 & 1758.66 & 2439.19 & 1909.14 & 1704.74 \\
\hline & Std.Dev & 38.44 & 67.68 & 331.22 & 115.41 & 120.83 \\
\hline \multirow{3}{*}{ eil101 } & Best & 677.08 & 820.46 & 1349.8 & 799.22 & 753.88 \\
\hline & Mean & 706.12 & 879.62 & 1502.71 & 888.44 & 821.606 \\
\hline & Std.Dev & 17.86 & 35.36 & 81.54 & 48.58 & 41.08 \\
\hline \multirow{3}{*}{$\operatorname{lin} 105$} & Best & 14866 & 18886 & 18835 & 22716 & 21742 \\
\hline & Mean & 15775.3 & 21528.13 & 24850.57 & 26261.37 & 24559.77 \\
\hline & Std.Dev & 435.41 & 1364.24 & 3183.79 & 1823.45 & 2115.15 \\
\hline \multirow{3}{*}{ bier127 } & Best & 124550 & 165840 & 294650 & 18892 & 154490 \\
\hline & Mean & 133512.3 & 181364.67 & 333406.67 & 168639.4 & 167539.67 \\
\hline & Std.Dev & 3491.55 & 7036.82 & 14019.24 & 29928.47 & 7540.24 \\
\hline \multirow{3}{*}{$\operatorname{ch} 130$} & Best & 6372.3 & 10029 & 21481 & 9339.2 & 8757.4 \\
\hline & Mean & 6936.59 & 10959.5 & 24078.37 & 10414.54 & 9860.80 \\
\hline & Std.Dev & 200.00 & 661.58 & 1063.32 & 719.059 & 529.52 \\
\hline \multirow{3}{*}{$\operatorname{ch} 150$} & Best & 7047.9 & 12626 & 19775 & 10500 & 9875.6 \\
\hline & Mean & 7558.40 & 13598.3 & 24356.17 & 11882.33 & 11085.08 \\
\hline & Std.Dev & 239.26 & 672.58 & 3774.56 & 725.86 & 611.69 \\
\hline
\end{tabular}

the results of the experimental results listed in Table 3, genetic operators (probability initialization, probabilistic crossover, deterministic crossover, and directional mutation operator) play a positive role on IHPSO.

\section{CONCLUSION}

In this paper, we proposed an IHPSO to deal with TSP. In IHPSO, there are four novel strategies are introduced to improve its performance. Firstly, a probability initialization method rather than the popular randomly initialization is introduced to improve the population's initial fitness. Secondly, a probabilistic crossover operator and a deterministic crossover operator are conducted between an individual and its Pbest and the Gbest. In this condition, useful information with Pbest and Gbest 
International Journal of Cognitive Informatics and Natural Intelligence

Volume 15 • Issue 4 • October-December 2021

Table 2. Comparison times(s) between IHPSO and 4 peer algorithms

\begin{tabular}{|c|c|c|c|c|c|c|}
\hline & & IHPSO & HPSO & GA & SA & TB \\
\hline eil51 & Mean & 458.12 & 336.76 & 1261.63 .90 & 1084.90 & 59.72 \\
\hline berlin52 & Mean & 441.00 & 345.12 & 9238.71 & 9276.67 & 58.93 \\
\hline eil76 & Mean & 587.47 & 600.76 & 2078.64 & 1571.83 & 64.38 \\
\hline rat99 & Mean & 777.94 & 928.10 & 2932.82 & 2013.41 & 63.02 \\
\hline eil101 & Mean & 806.36 & 1077.32 & 3353.38 & 2070.76 & 66.73 \\
\hline lin105 & Mean & 819.53 & 1028.60 & 3225.13 & 2143.11 & 64.47 \\
\hline bier127 & Mean & 1110.73 & 1758.83 & 4324.36 & 2563.58 & 63.25 \\
\hline ch130 & Mean & 1099.81 & 1504.10 & 4497.03 & 2606.47 & 64.56 \\
\hline ch150 & Mean & 1325.63 & 1959.64 & 5262.29 & 2982.99 & 69.17 \\
\hline \multicolumn{2}{|c|}{ Avg } & 825.18 & 1059.91 & 3140.34 & 2015.83 & 63.80 \\
\hline \multicolumn{2}{|c|}{ Ranking } & 2 & 3 & 5 & 4 & 1 \\
\hline
\end{tabular}

Figure 8. Tour path of the 9 benchmark problems

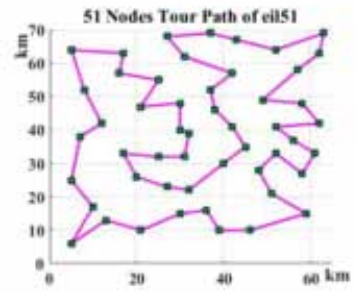

(a) eil51

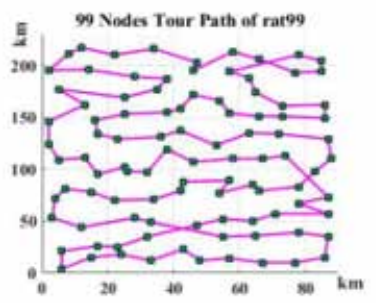

(d) rat99

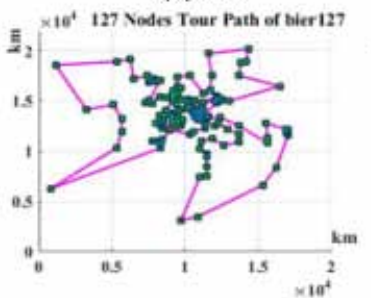

(g) bier127

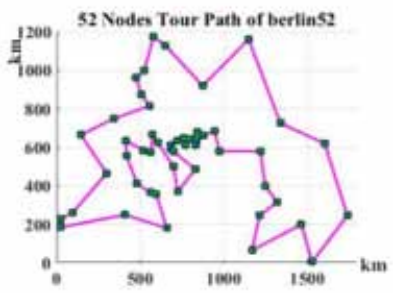

(b) berlin 52

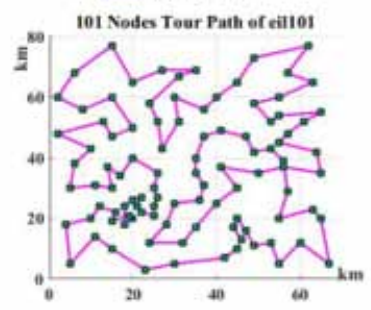

(e) eillo1

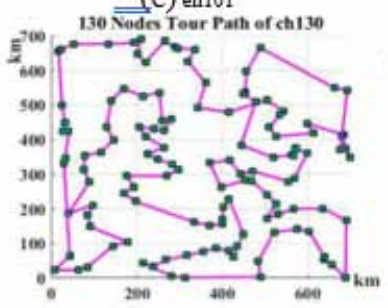

(h) ch130

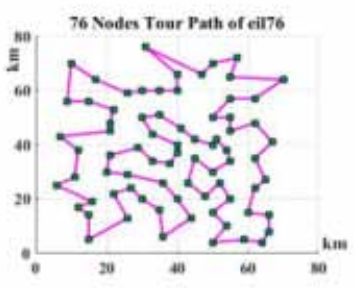

(c) eil76

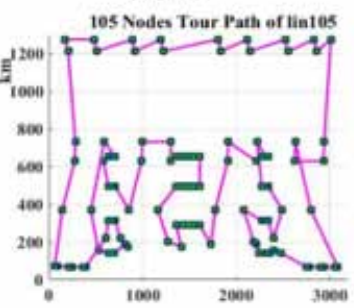

(f) $\operatorname{lin} 105$

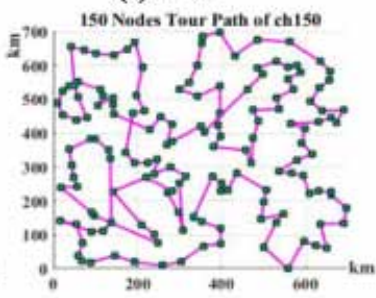

(i) $\operatorname{ch} 150$ 
Table 3. Comparison results(km) between IHPSO and IHPSO(a), IHPSO(b), and IHPSO(d)

\begin{tabular}{|c|c|c|c|c|c|c|}
\hline & & IHPSO & IHPSO(a) & IHPSO(b) & IHPSO(c) & $\operatorname{IHPSO}(d)$ \\
\hline \multirow{2}{*}{ eil51 } & Best & 428.87 & 433.64 & 437.15 & 480.48 & 434.15 \\
\hline & Mean & 448.35 & 450.25 & 451.32 & 450.13 & 450.12 \\
\hline \multirow{2}{*}{ berlin52 } & Best & 7544.4 & 7695.10 & 7616 & 8330.20 & 7620.10 \\
\hline & Mean & 7938.49 & 8089.25 & 8046.23 & 7973.32 & 8033.06 \\
\hline \multirow{2}{*}{ eil76 } & Best & $\mathbf{5 5 7 . 5 0}$ & 573.48 & 570.47 & 620.90 & 566.00 \\
\hline & Mean & 581.66 & 590.94 & 592.35 & 592.23 & 591.06 \\
\hline \multirow{2}{*}{ rat99 } & Best & 1249.8 & 1340.20 & 1284.50 & 1452.60 & 1289.40 \\
\hline & Mean & 1356.21 & 1417.04 & 1390.43 & 1393.35 & 1372.15 \\
\hline \multirow{2}{*}{ eil101 } & Best & 677.08 & 689.50 & 680.06 & 777.32 & 693.3943 \\
\hline & Mean & 706.12 & 726.69 & 719.58 & 729.15 & 724.01 \\
\hline \multirow{2}{*}{$\operatorname{lin} 105$} & Best & 14866 & 15469 & 15239 & 16660 & 14952 \\
\hline & Mean & 15775.3 & 17048.47 & 16007.07 & 15773.87 & 15873 \\
\hline \multirow{2}{*}{ bier127 } & Best & 124550 & 132240 & 128980 & 144350 & 129980 \\
\hline & Mean & 133512.3 & 138012.33 & 134067 & 135979 & 138055 \\
\hline \multirow{2}{*}{$\operatorname{ch} 130$} & Best & 6372.3 & 7131.40 & 6736.30 & 7563.30 & 6798.10 \\
\hline & Mean & 6936.59 & 7639.97 & 7139.12 & 7060.04 & 7186.39 \\
\hline \multirow{2}{*}{$\operatorname{ch} 150$} & Best & 7047.9 & 7888.70 & 7428.60 & 8156.40 & 7322.50 \\
\hline & Mean & 7558.40 & 8723.49 & 7734.69 & 7697.49 & 7824.04 \\
\hline
\end{tabular}

can be effectively utilized to balance the convergence speed and population diversity. Lastly, a directional mutation operator is adopted to add a little disturbance to improve population diversity. The comparison results between IHPSO and the other four types of EAs on nine TSP test problems to verify the outstanding performance of IHPSO. In addition, compared to the canonical PSO, IHPSO displays more favorable performance on large scale TSP than on small size TSP.

\section{ACKNOWLEDGMENT}

This study was funded by the National Natural Science Foundation of China [Grant Nos.: 61806204, 61663009, 61602174], the National Natural Science Foundation of Jiangxi Province[Grant Nos.: 20171BAB202019, 20161BAB202064, 20161BAB212052, 20151BAB207022], the National Natural Science Foundation of Jiangxi Provincial Department of Education [Grant No.: GJJ160469], and the Research Project of Jiangxi Provincial Department of Communication and Transportation [Grant No. 2017D0038]. 


\section{REFERENCES}

Bello, R., Gomez, Y., \& Nowe, A. (2007). Two-step particle swarm optimization to solve the feature selection problem. In 7th International Conference on Intelligent Systems Design and Applications(pp. 691-696). Atlanta, GA: IEEE. doi:10.1109/ISDA.2007.101

Chandramouli, K., \& Izquierdo, E. (2006). Image classification using chaotic particle swarm optimization. In 8th International Conference on Image Processing (pp. 3001-3004). Atlanta, GA: IEEE. doi:10.1109/ ICIP.2006.312968

Chang, J. F. (2005). A parallel particle swarm optimization algorithm with communication strategies. Journal of Information Science and Engineering, 21(4), 809-818.

Chatterjee, A., Pulasinghe, K., Watanabe, K., \& Izumi, K. (2005). A particle-swarm-optimized fuzzy-neural network for voice-controlled robot systems. IEEE Transactions on Industrial Electronics, 52(6), 1478-1489. doi:10.1109/TIE.2005.858737

Chen, J. F., Ren, Z. W., \& Fan, X. N. (2006). Particle swarm optimization with adaptive mutation and its application research in tuning of PID parameters. In 1th International Symposium on Systems and Control in Aerospace and Astronautics (vol. 26, pp. 1-5). Atlanta, GA: IEEE.

Clerc, M. (2004). Discrete particle swarm optimization, illustrated by the traveling salesman problem. In New Optimization Techniques in Engineering. Springer.

Hendtlass, T. (2003). Preserving diversity in particle swarm optimization. Lecture Notes in Computer Science, 2718, 4104-4108. doi:10.1007/3-540-45034-3_4

Kennedy, J., \& Eberhart, R. C. (1995). Particle swarm optimization. In Proceedings of IEEE International Conference on Neural Network, ICNN'95 (vol. 4, pp.1942-1948). Perth, Australia: IEEE. doi:10.1109/ ICNN.1995.488968

Li, K. S., Chen, Y., Li, W., He, J., \& Xue, Y. (2018). Improved gene expression programming to solve the inverse problem for ordinary differential equations. Swarm and Evolutionary Computation, 38, 231-239. doi:10.1016/j. swevo.2017.07.005

Li, K. S., Liang, Z. Z., Yang, S., Chen, Z., Wang, H., \& Lin, Z. (2019). Performance analyses of differential evolution algorithm based on dynamic fitness landscape. International Journal of Cognitive Informatics and Natural Intelligence, 13(1), 36-61. doi:10.4018/IJCINI.2019010104

Li, K. S., Wang, H., \& Li, S. (2019). A mobile node localization algorithm based on an overlapping self-adjustment mechanism. Information Sciences, 481, 635-649. doi:10.1016/j.ins.2018.12.006

Li, R., Qiao, J., \& Li, W. (2016). A modified hopfield neural network for solving TSP problem. In 12th World Congress on Intelligent Control and Automation (pp. 1775-1780). Guilin, China: IEEE. doi:10.1109/ WCICA.2016.7578744

Li, Y., Zhou, A., \& Zhang, G. (2011). Simulated annealing with probabilistic neighborhood for traveling salesman problems. In 7th International Conference on Natural Computation (pp. 1565-1569). Shanghai, China: IEEE. doi:10.1109/ICNC.2011.6022345

Liao, Y. F., Yau, D. H., \& Chen, C. L. (2012). Evolutionary algorithm to traveling salesman problems. Computers \& Mathematics with Applications (Oxford, England), 64(5), 788-797. doi:10.1016/j.camwa.2011.12.018

Lin, Y., Bian, Z., \& Liu, X. (2016). Developing a dynamic neighborhood structure for an adaptive hybrid simulated annealing - tabu search algorithm to solve the symmetrical traveling salesman problem. Applied Soft Computing, 49, 937-952. doi:10.1016/j.asoc.2016.08.036

Ling, S. H., Iu, H. H., Chan, K. Y., Lam, H. K., Yeung, B. C. W., \& Leung, F. H. (2008). Hybrid particle swarm optimization with wavelet mutation and its industrial applications. IEEE Transactions on Cybernetics, 38(3), 743-763. doi:10.1109/TSMCB.2008.921005 PMID:18558539

Pang, W., Wang, K. P., \& Zhou, C. G. (2004). Fuzzy discrete particle swarm optimization for solving traveling salesman problem. In The 4th International Conference on Computer and Information Technology (vol.3, pp. 796-800). Beijing, China: IEEE. 
Pang, W., Wang, K. P., Zhou, C. G., \& Dong, L. J. (2004). Fuzzy discrete particle swarm optimization for solving traveling salesman problem. In 4th International Conference on Computer and Information Technology (vol. 3 , pp. 796-800). Wuhan, China: IEEE.

Reinelt, G. (1991). TSPLIB-A traveling salesman problem library. ORSA Journal on Computing, 3(4), 376-384. doi:10.1287/ijoc.3.4.376

Shaj, V., Akhil, P. M., \& Asharaf, S. (2016). Edge-PSO: A recombination operator based PSO algorithm for solving TSP, In 6th International Conference on Advances in Computing, Communications and Informatics(pp. 35-41). Kochi, India: IEEE. doi:10.1109/ICACCI.2016.7732022

Wang, F., Zhang, H., Li, K. S., Lin, Z. Y., Yang, J., \& Shen, X.-L. (2018). A hybrid particle swarm optimization algorithm using adaptive learning strategy. Information Sciences, 436-437, 162-177. doi:10.1016/j.ins.2018.01.027

Wang, K. P., Huang, N. L., \& Zhou, C. G. (2003). Particle swarm optimization for traveling salesman problem. Acta Scientiarium Naturalium Universitatis Jilinensis, 3(4), 1583-1585.

Yin, L., Li, X., \& Gao, L. (2016). A new improved fruit fly optimization algorithm for traveling salesman problem. In: 8th International Conference on Advanced Computational Intelligence (pp. 21-28). Chiang Mai, Thailand: IEEE. doi:10.1109/ICACI.2016.7449797

Bo Wei received the Ph.D. degree in Computer Software and Theory from Wuhan University, Wuhan, China, in 2013. He is currently a Lecturer with the School of Information Science and Technology, Zhejiang Sci-Tech University, China. In 2015, he worked as a postdoctoral researcher at Institute of Automation, Chinese Academy of Science, Beijing, China. His current research interests include the areas of computational intelligence techniques, machine learning, and data mining. 